\title{
Bioethica
}

Vol 6, No 2 (2020)

Bioethica

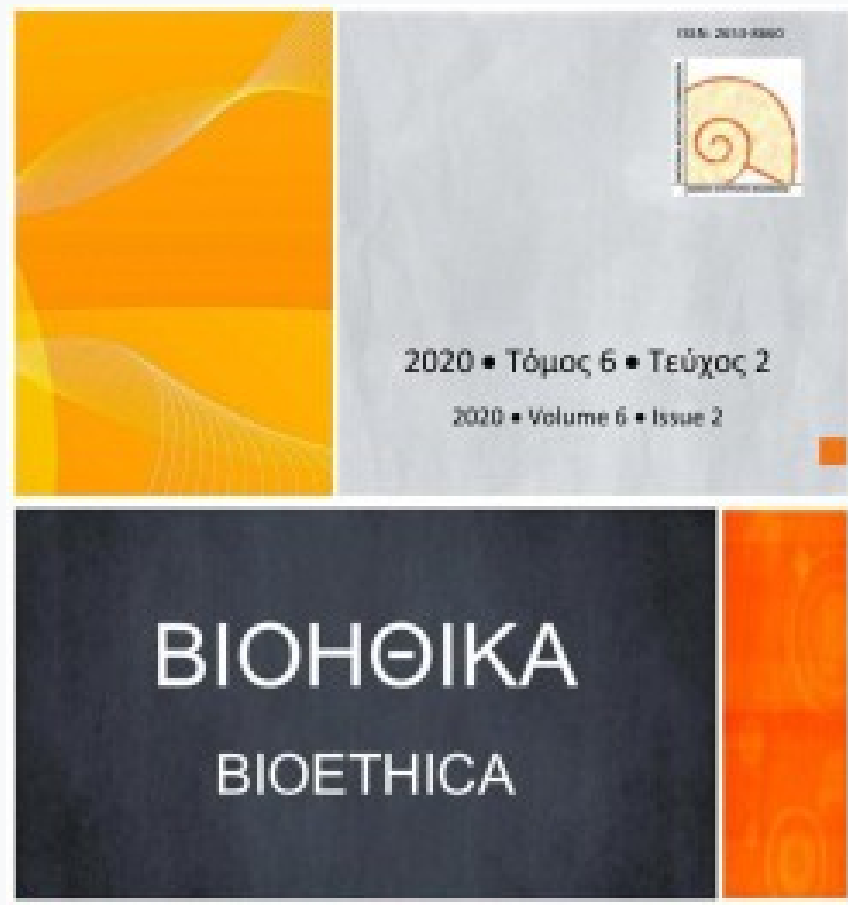

\section{Inventions for SARS-CoV-2 vaccines}

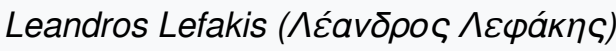

doi: $10.12681 /$ bioeth.24841

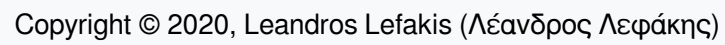

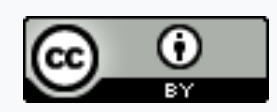

This work is licensed under a Creative Commons Attribution 4.0.

To cite this article:

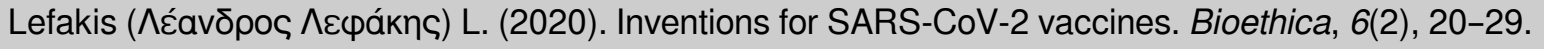

https://doi.org/10.12681/bioeth.24841 


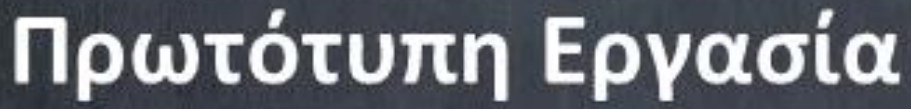

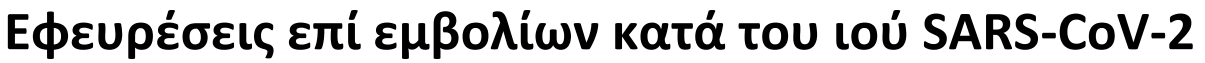

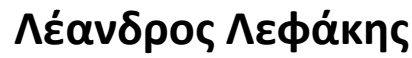

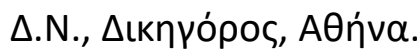

\section{leandros@lefakislaw.gr}

\section{Пєрí $\eta \psi \eta$}

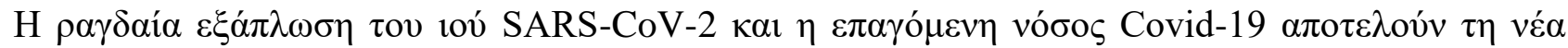

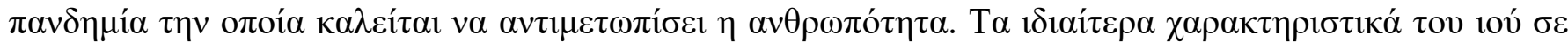

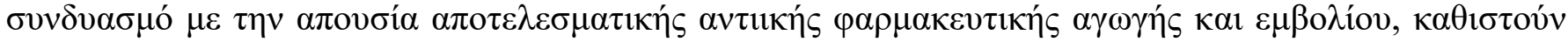

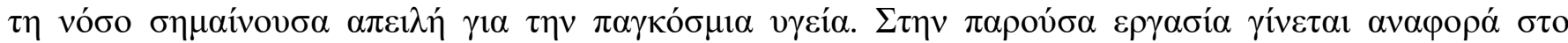

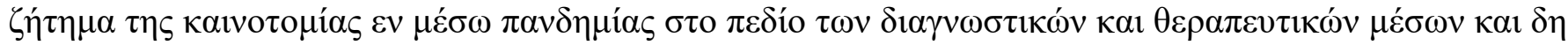

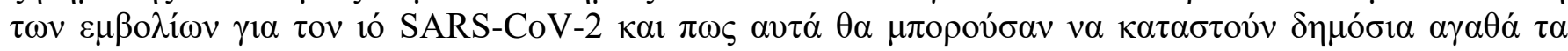

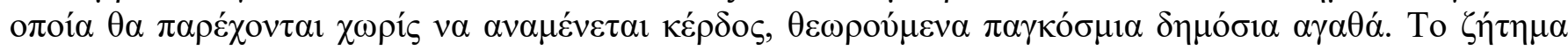

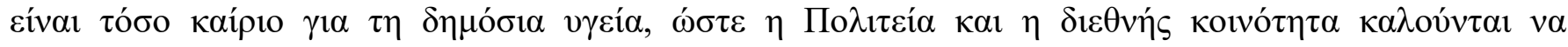

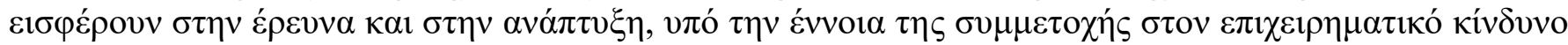

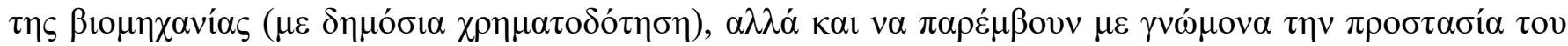

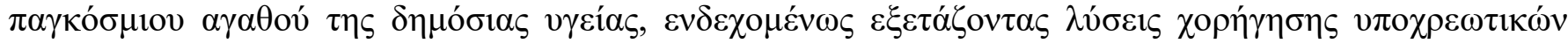

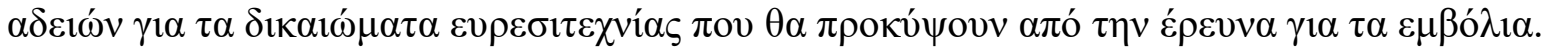

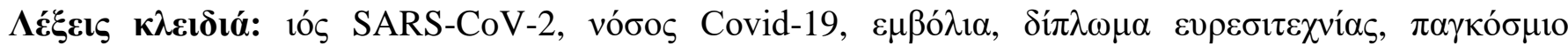

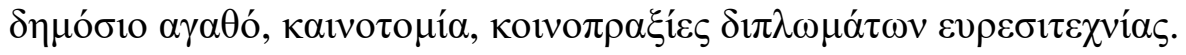




\title{
Inventions for SARS-CoV-2 vaccines
}

\section{Dr Leandros Lefakis}

Attorney at law, LLM, Greece.

\begin{abstract}
The rapid spread of the SARS-CoV-2 virus is the new pandemic that humanity is called upon to deal with. The special characteristics of the virus in combination with the absence of effective antiviral medication and vaccine, make the disease a significant threat to global health. This paper addresses the issue of innovation in the midst of a pandemic in the field of diagnostic and therapeutic procedures, namely vaccines for the SARS-CoV-2 virus and how these could become public goods that will be provided without expected profit, considered global public goods. The issue is so crucial for public health that the State and the international community are called upon to contribute to research and development, in terms of participating in the business risk of the industry (with public funding), but also to intervene to protection of the global good of public health, possibly considering compulsory patent licensing solutions that will result from vaccine research.
\end{abstract}

Keywords: SARS-CoV-2, Covid-19, patents, vaccines, global public good, innovation, patent pools. 


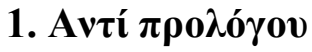

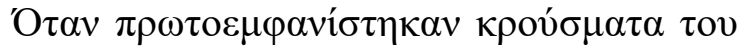
เov́ SARS-CoV-2 (Severe Acute Respiratory

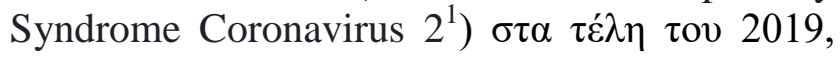

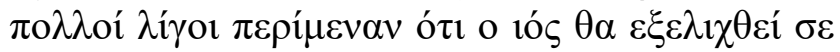

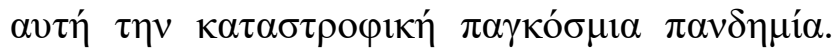

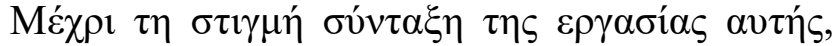

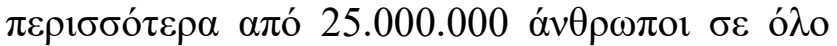

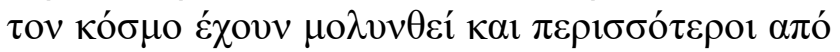

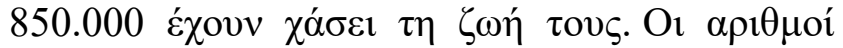

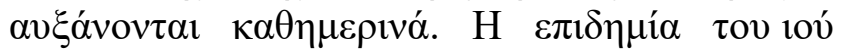

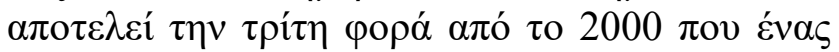

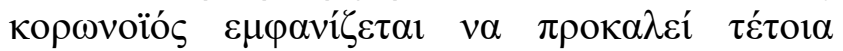

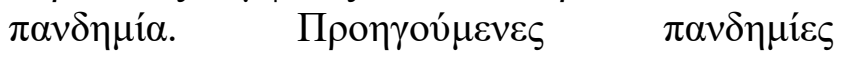

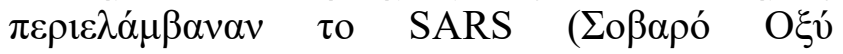

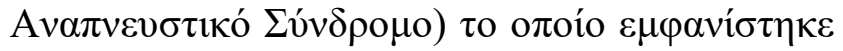

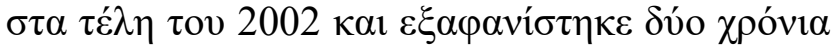

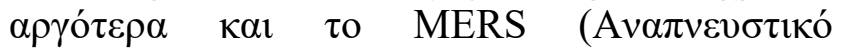

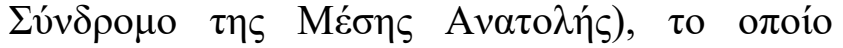

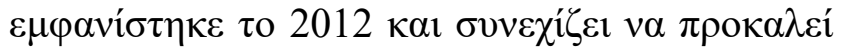

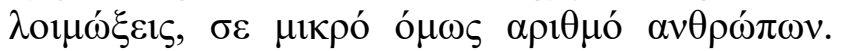

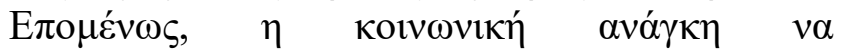

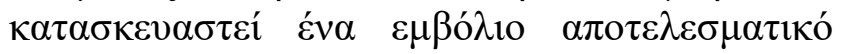

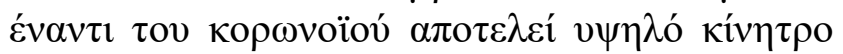

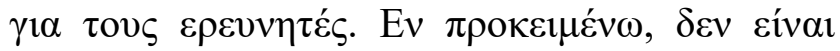

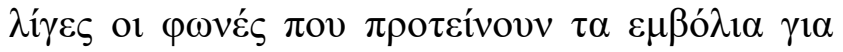

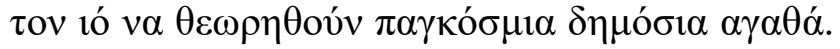

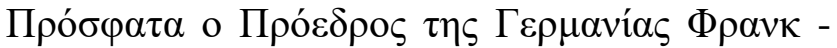

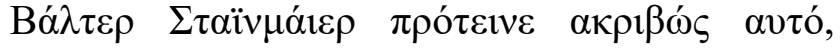

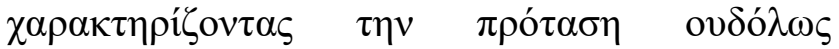

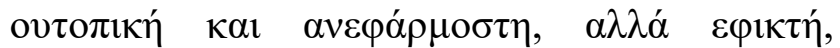

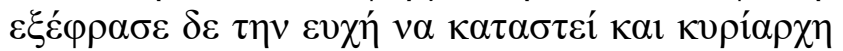
$\delta 1 \varepsilon \theta v \omega ́ s$.

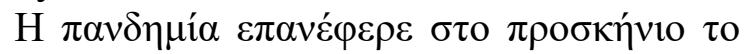
$\zeta \eta ́ \tau \eta \mu \alpha \quad \tau \eta \varsigma \quad \chi 0 \rho \eta ́ \gamma \eta \sigma \eta \varsigma \quad \delta 1 \kappa \alpha 1 \omega \mu \alpha ́ \tau \omega v$

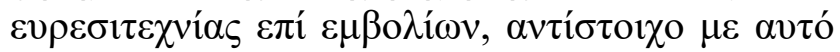

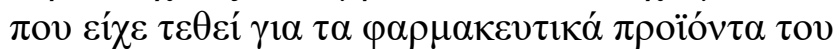

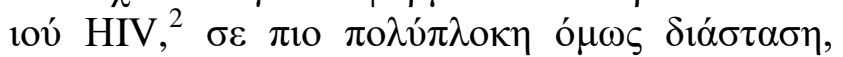

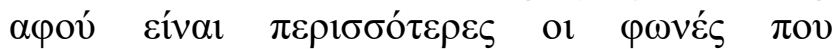

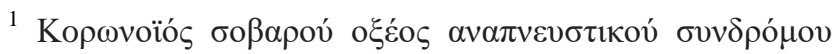

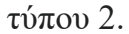

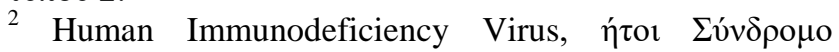

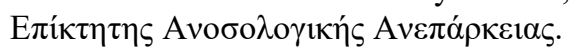

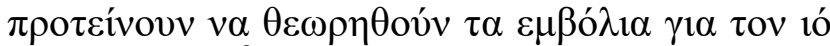
SARS-CoV-2 $2^{3} \pi \alpha \gamma \kappa o ́ \sigma \mu 1 \alpha \quad \delta \eta \mu o ́ \sigma ı \alpha ~ \alpha \gamma \alpha \theta \alpha ́$. H

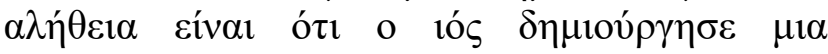

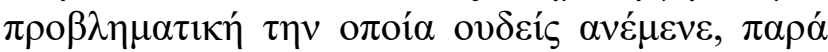

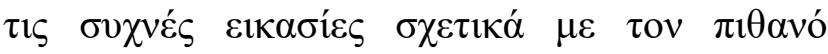

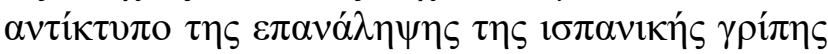

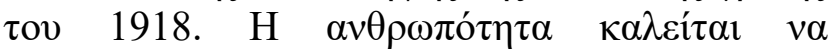

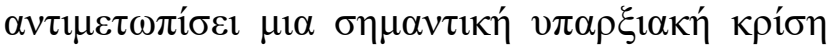

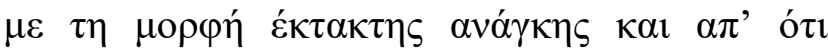

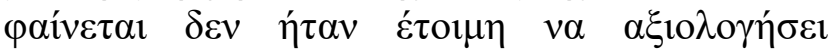

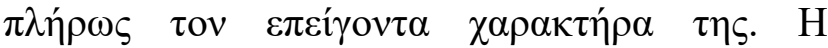

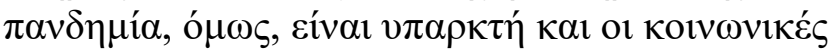

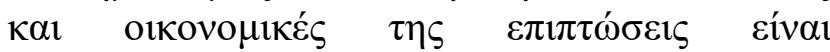

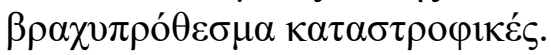

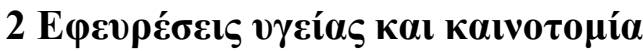

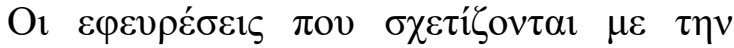

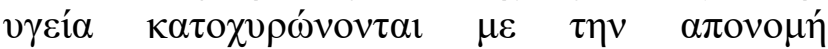

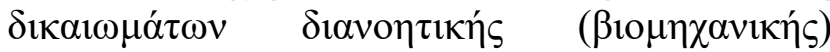

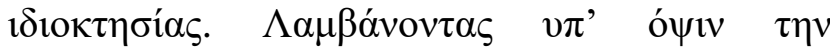

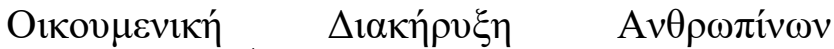

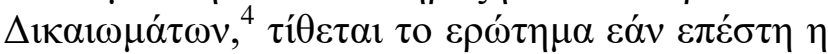

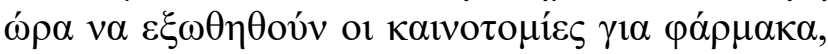

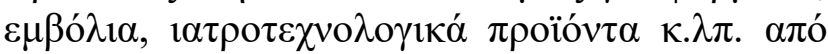

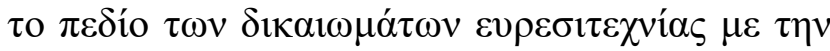

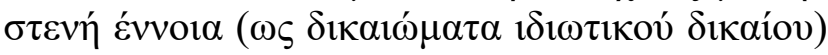

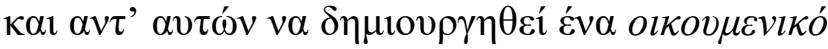

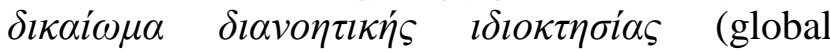

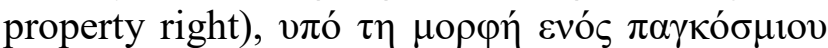

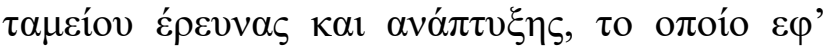

\footnotetext{
${ }^{3}$ Severe Acute Respiratory Syndrome Coronavirus 2, ท́

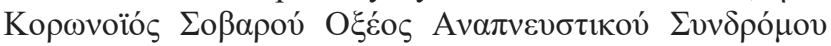
Tи́лov 2.

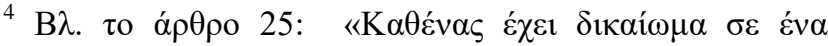

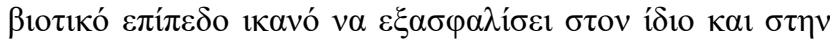

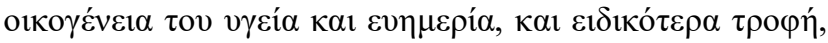

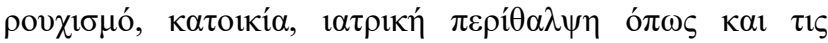

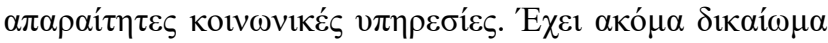

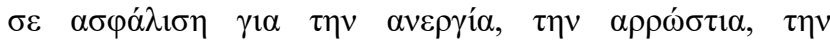

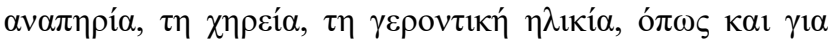

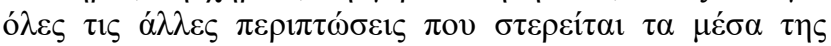

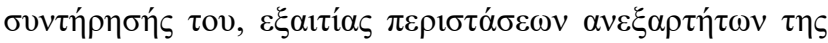

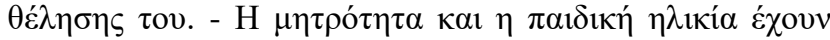

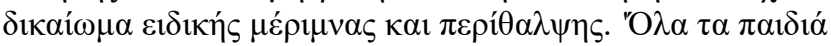

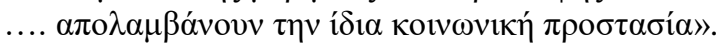




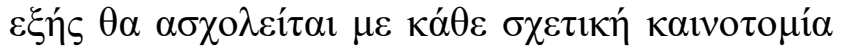

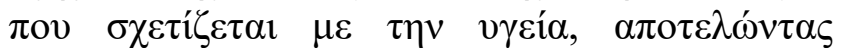

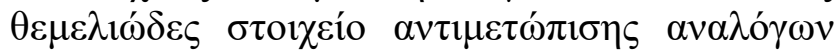

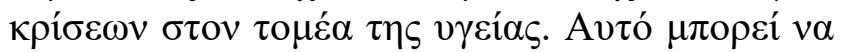

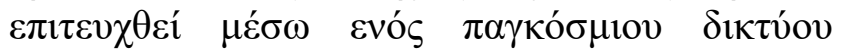

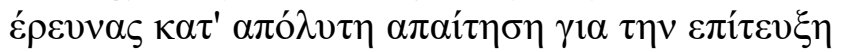

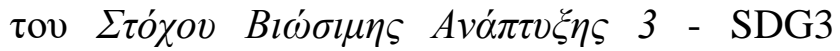

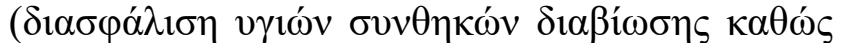

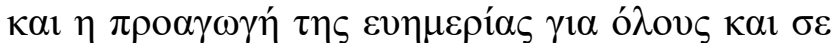

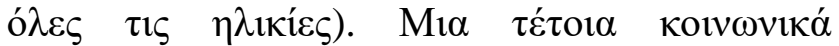

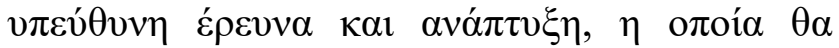

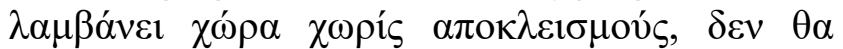

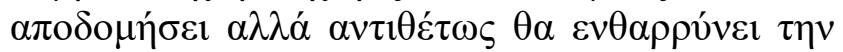

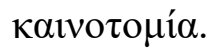

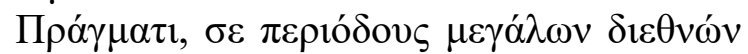

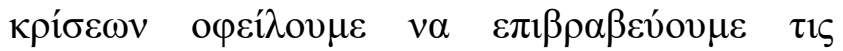

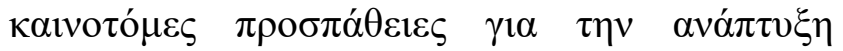

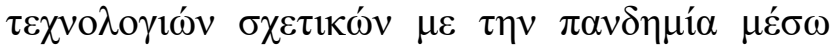

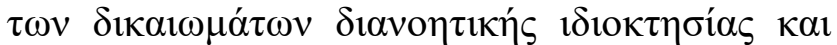

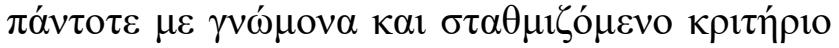

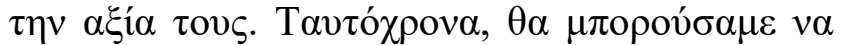
$\varepsilon \xi \alpha \sigma \varphi \alpha \lambda i ́ \sigma o v \mu \varepsilon \tau \eta \eta$

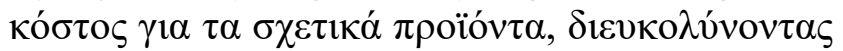

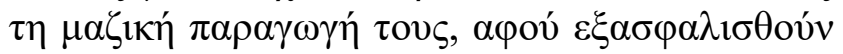

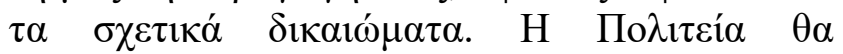

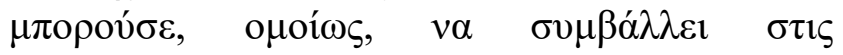

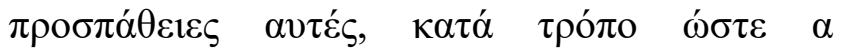

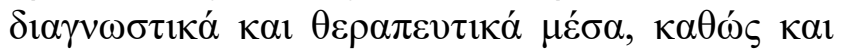

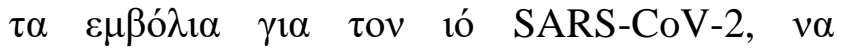

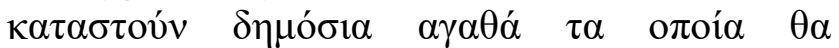

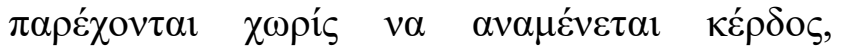

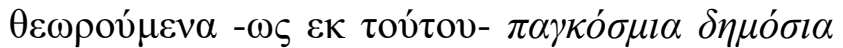

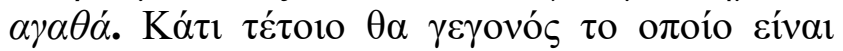

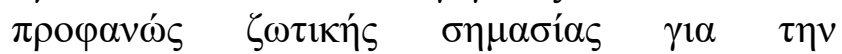

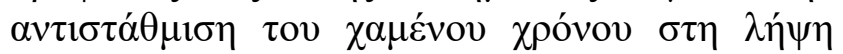

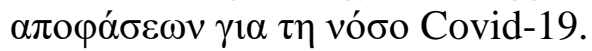

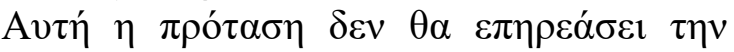

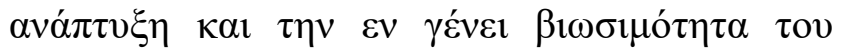

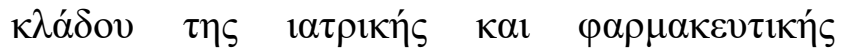

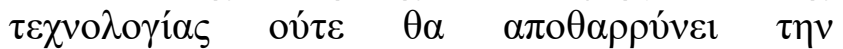

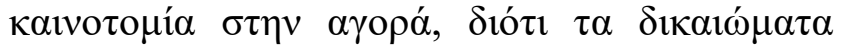

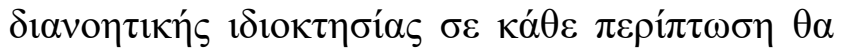

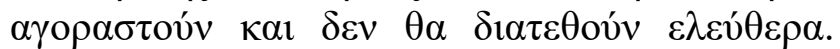

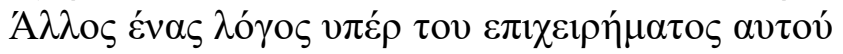

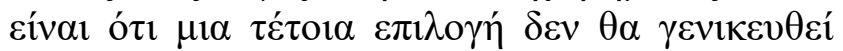

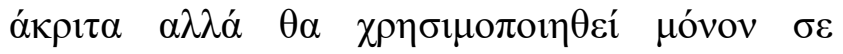

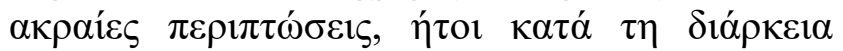

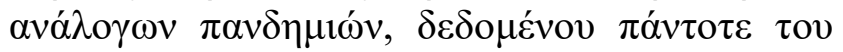

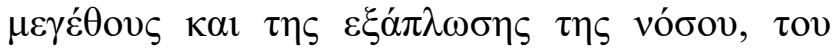

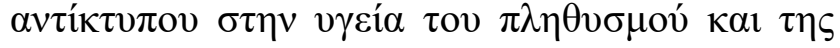

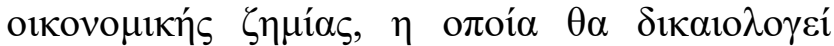

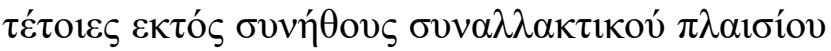

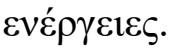

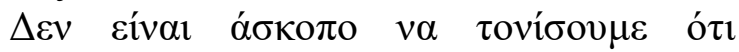

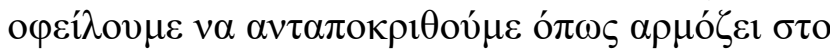

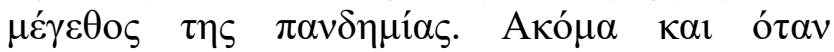

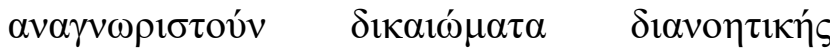

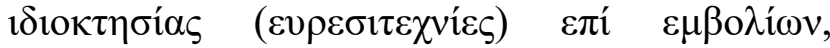

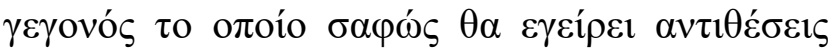

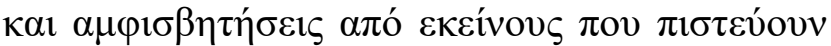

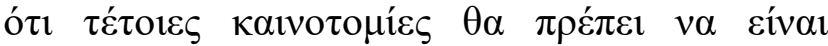

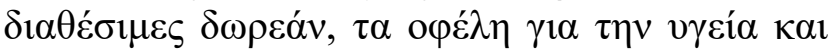

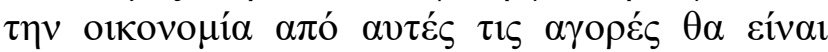

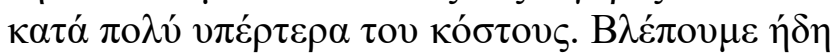

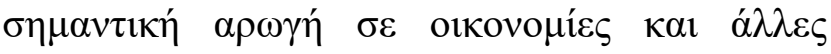

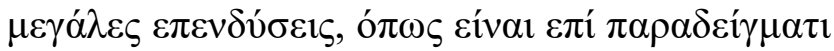

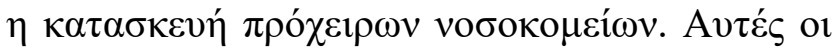

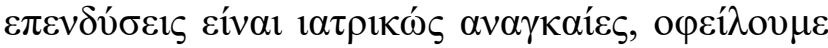

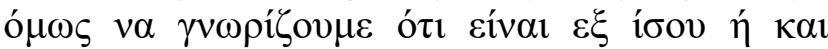

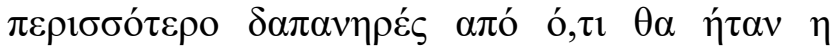

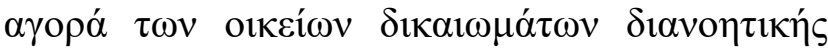

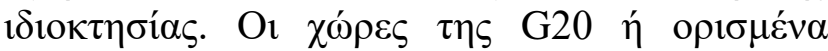

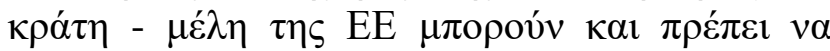

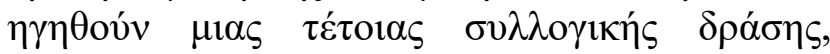

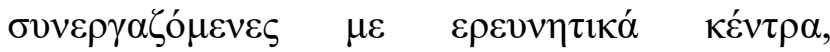

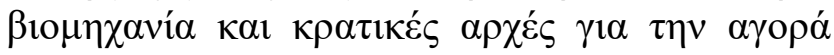

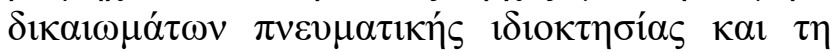

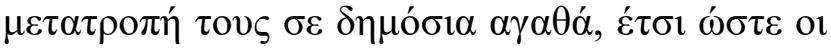

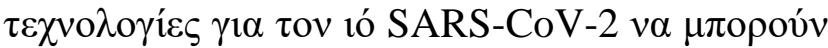

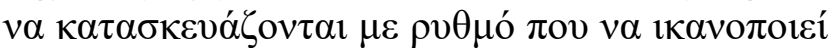

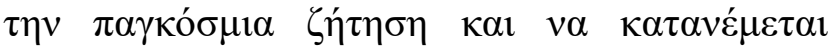

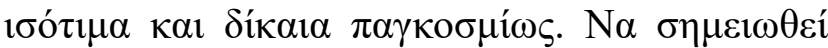

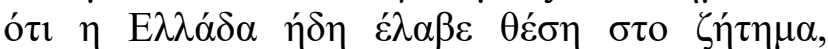

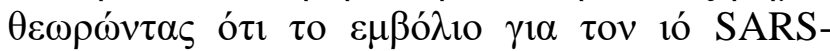

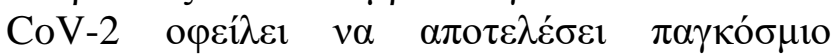

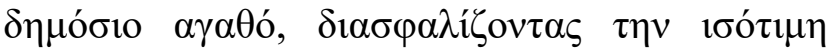
$\pi \rho o ́ \sigma \beta \alpha \sigma \eta$ ó $\lambda \omega v \tau \omega v \chi \omega \rho \omega ́ v ~ \sigma \tau \eta v ~ \kappa \alpha \tau \alpha \pi \circ \lambda \varepsilon \dot{\varepsilon} \mu \eta \sigma \eta$ $\tau \eta \varsigma \pi \alpha v \delta \eta \mu i ́ \alpha \varsigma$.

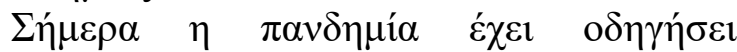
o

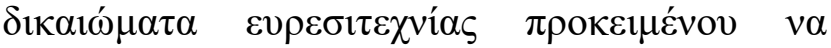

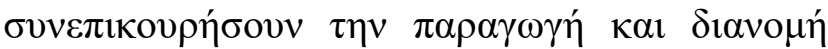
$\varepsilon \mu \beta о \lambda i ́ \omega v, \theta \varepsilon \rho \alpha \pi \varepsilon i \omega ́ v, \delta 1 \alpha \gamma v \omega \sigma \tau 1 \kappa \omega ́ v \varepsilon \xi \varepsilon \tau \alpha \dot{\sigma} \sigma \omega v$

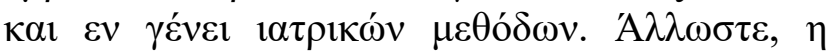

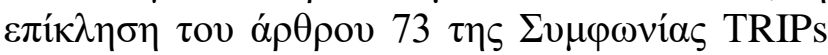

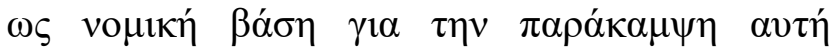

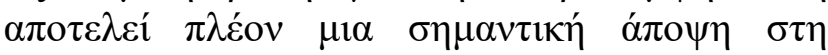

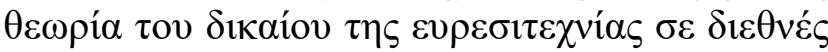

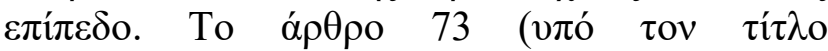

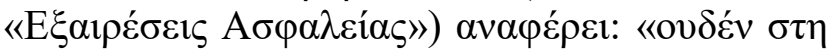




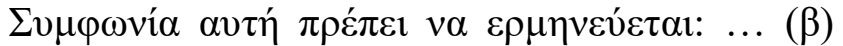

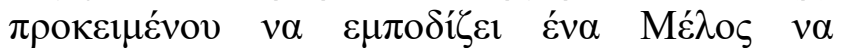

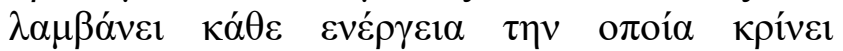

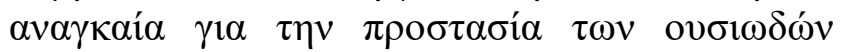

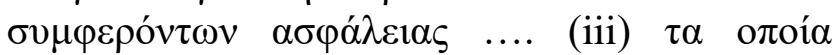

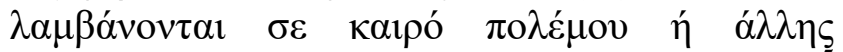

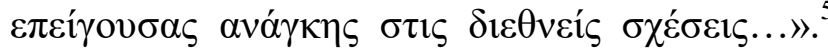

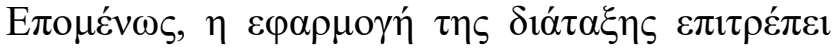

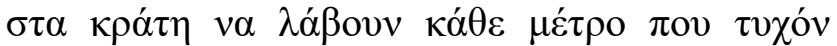

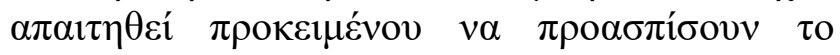

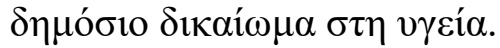

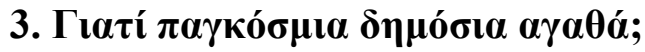

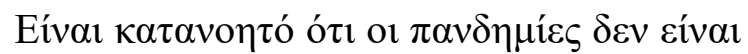

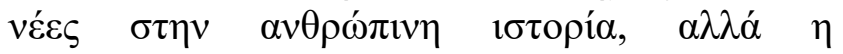

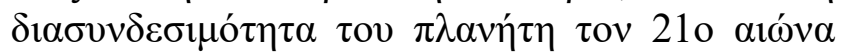

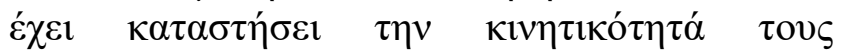

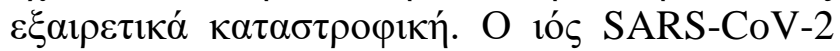

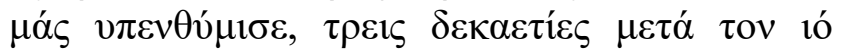

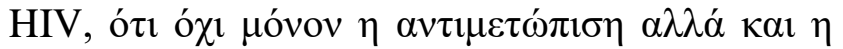
$\pi \rho o ́ \lambda \eta \psi \eta \quad \alpha \sigma \theta \varepsilon v \varepsilon 1 \omega ́ v \quad \pi \rho \varepsilon ́ \pi \varepsilon 1 \quad v \alpha \quad \alpha \pi 0 \tau \varepsilon \lambda \varepsilon \dot{\sigma \varepsilon \varepsilon}$

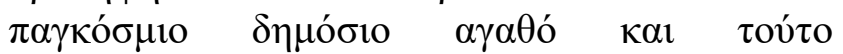

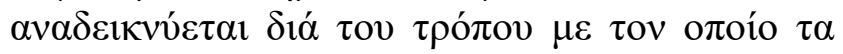

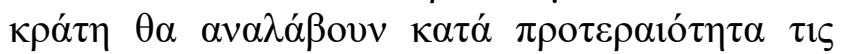

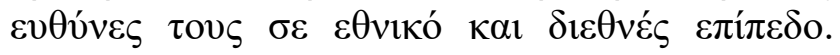

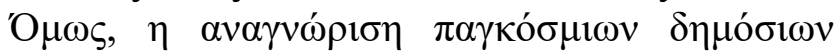

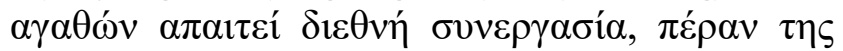
$\delta i \alpha \mu \varepsilon \sigma o \lambda \alpha ́ \beta \eta \sigma \eta \varsigma \tau \omega v \delta i \varepsilon \theta v \omega ́ v$ o $\gamma \alpha \alpha v i \sigma \mu \omega ́ v$.

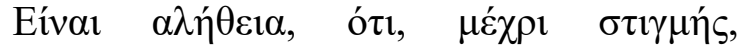

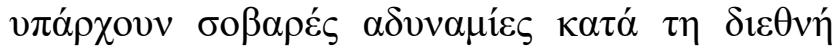

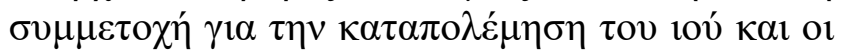

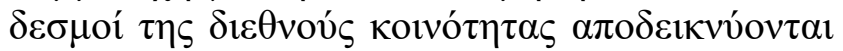

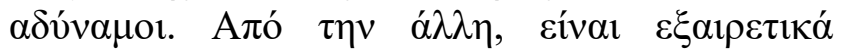

\footnotetext{
${ }^{5}$ B $\lambda$. Abbott F, The TRIPs Agreement Article 73 Security Exceptions and the COVID-19 Pandemic, Research Paper

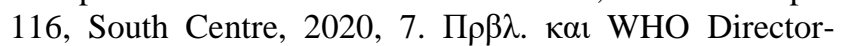
General's statement on IHR Emergency Committee on Novel Coronavirus (2019-nCoV), 30 Jan. 2020. https://www.who.int/. П $\rho \beta \lambda$. $\kappa \alpha$ l Branswell H, As coronavirus pandemic worsens, health officials fear nationalization of drugs and supplies, Stat Health, 15 March 2020, https://www.statnews.com/2020/03/15/ascoronavirus-pandemic-worsens-health-officials-fearnationalization-of-drugs-and-supplies.
}

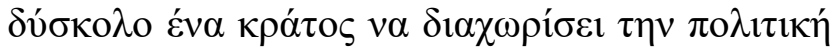

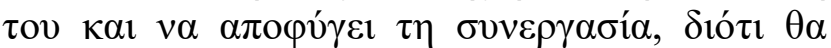

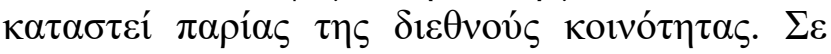

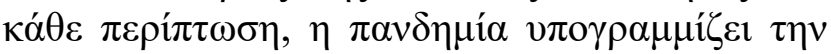
$\varepsilon \pi \imath \tau \alpha \kappa \tau \imath \kappa \eta ́ ~ \alpha v \alpha ́ \gamma \kappa \eta ~ v \alpha \alpha v \alpha \gamma v \omega \rho \iota \sigma \tau \varepsilon i ́ ~ \eta ~ \varepsilon \pi \imath v v \mu \eta \tau \eta ́$ $\alpha \lambda \lambda \eta \lambda \varepsilon \xi \alpha \dot{\alpha} \rho \tau \eta \sigma \eta \quad \gamma 1 \alpha \quad \tau \eta v \quad \varepsilon \xi \varepsilon v ́ \rho \varepsilon \sigma \eta \quad \lambda v ́ \sigma \varepsilon \omega v$

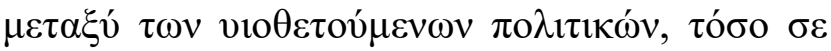

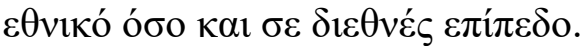

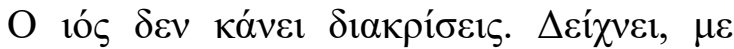

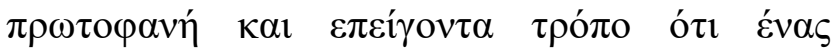

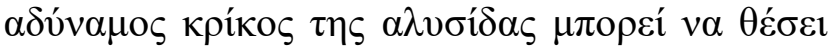

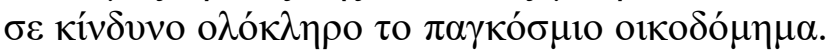

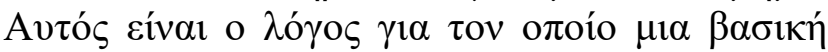

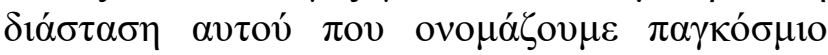

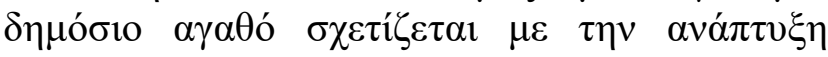

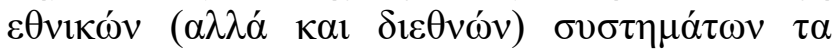

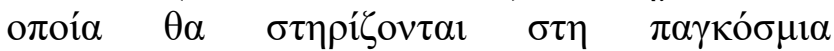

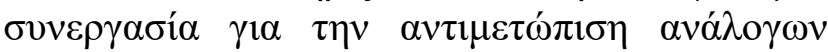
$\alpha \pi \varepsilon \imath \lambda \omega ́ v$.

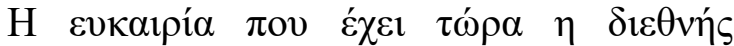

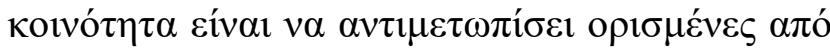
$\tau ı \varsigma \sigma v \sigma \tau \eta \mu 1 \kappa \varepsilon \dot{\varepsilon} \varsigma \alpha \delta v v \alpha \mu i ́ \varepsilon \varsigma \tau \omega v \delta 1 \varepsilon \theta v \omega ́ v \theta \varepsilon \sigma \mu \omega ́ v$,

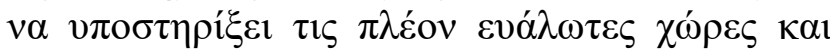

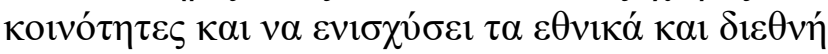

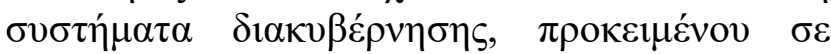

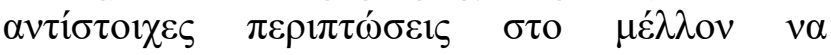

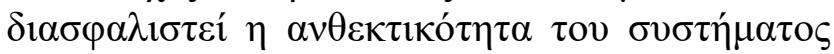
vүcías.

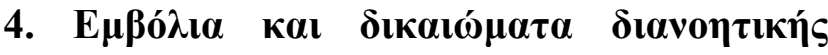

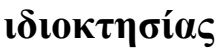

$\Sigma v ́ \mu \varphi \omega v \alpha \mu \varepsilon \tau \eta$ STAT, or $\pi \rho \circ \sigma \pi \alpha ́ \theta \varepsilon 1 \varepsilon \varsigma \tau \omega v$

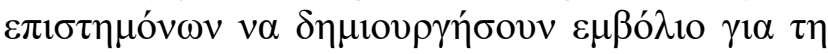

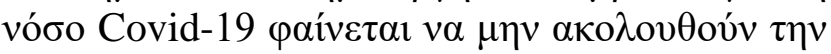

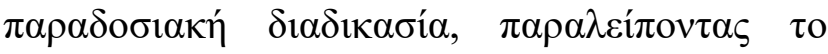

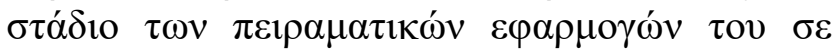
$\zeta \omega ́ \alpha$. 'О

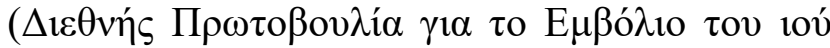

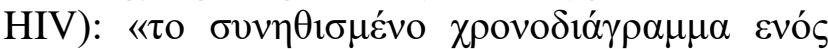

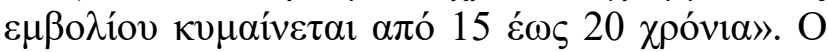

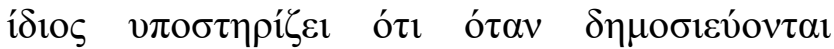

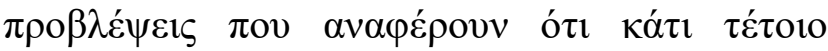

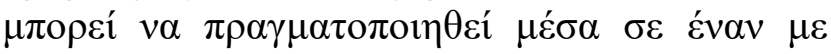

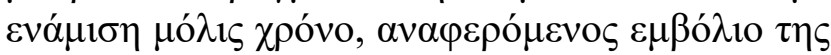

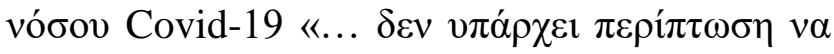

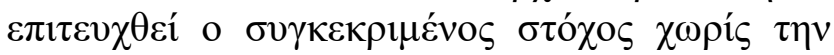

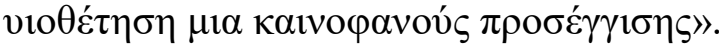

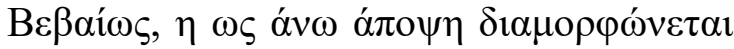

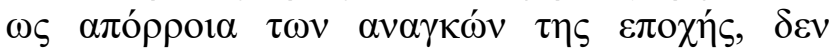




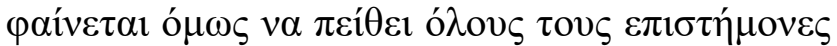

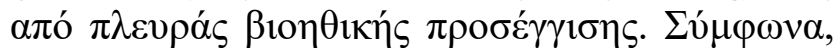

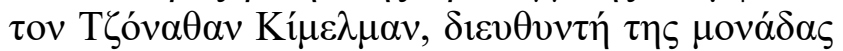

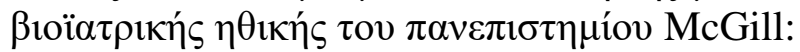

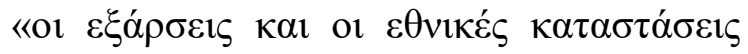

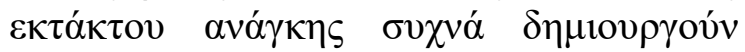

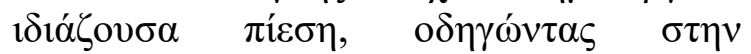

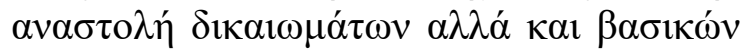

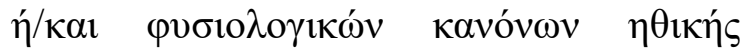

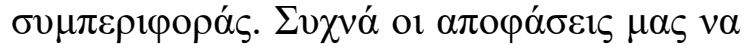

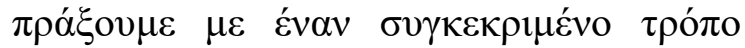

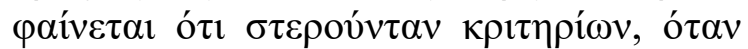

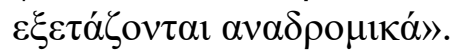

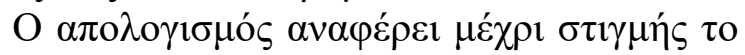

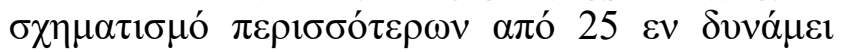

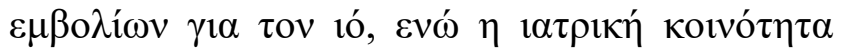

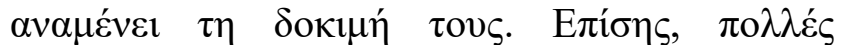

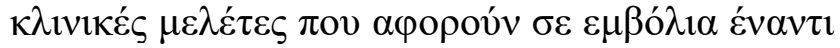

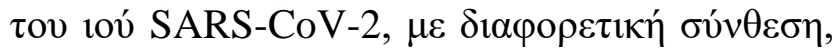

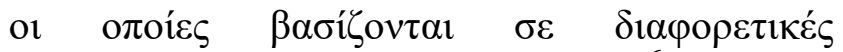

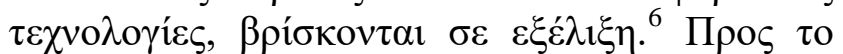

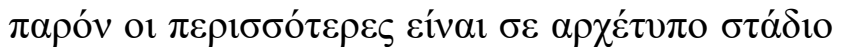

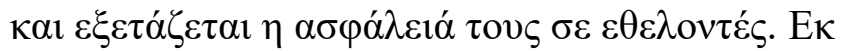

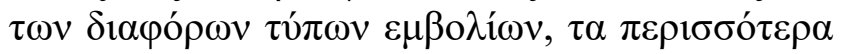

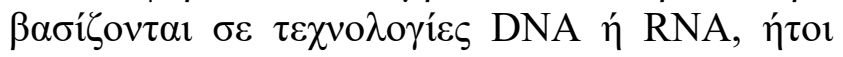

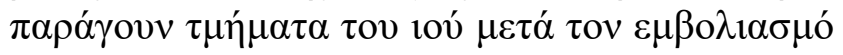

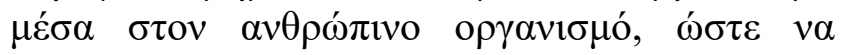

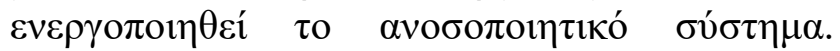

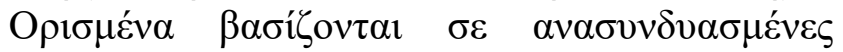

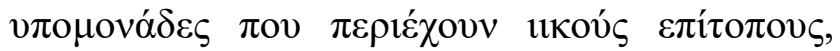

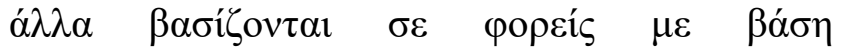

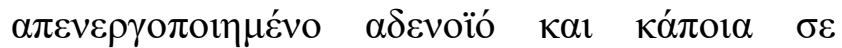

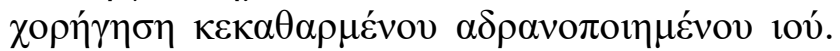

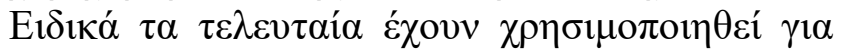

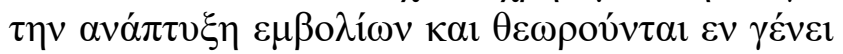

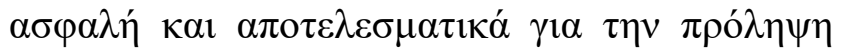

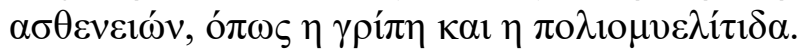

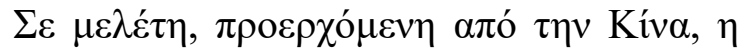

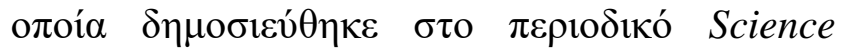

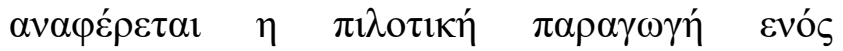

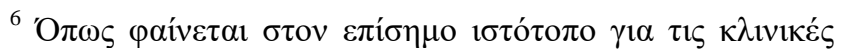
$\mu \varepsilon \lambda \varepsilon ́ \tau \varepsilon \zeta$ (https://www.clinicaltrials.gov/).
}

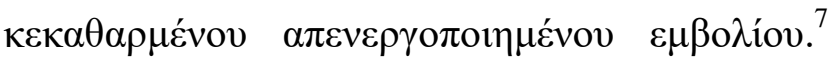

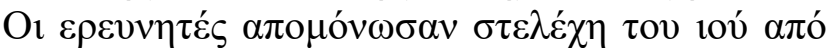

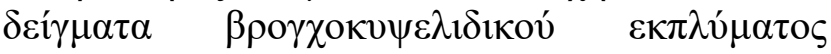

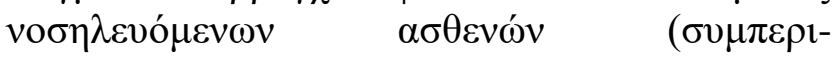

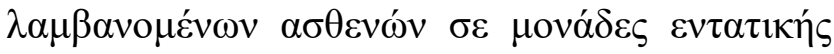

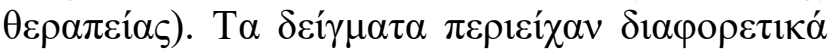

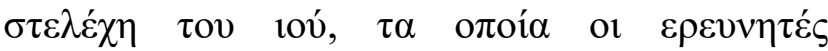

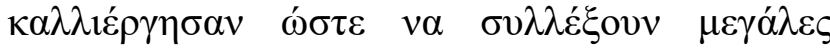

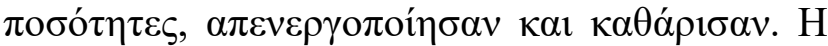

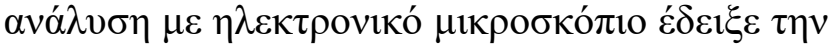

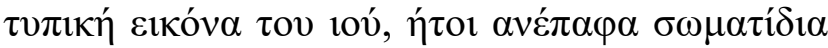

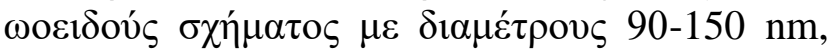

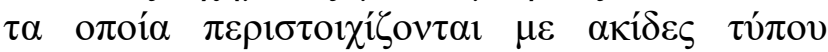

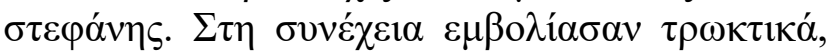

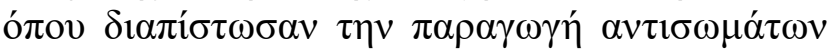

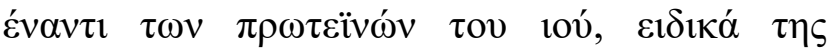

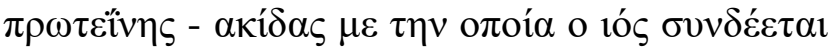

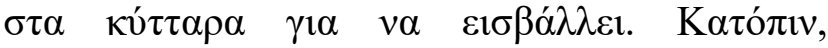

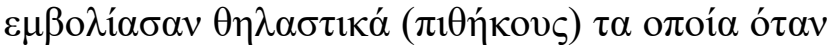

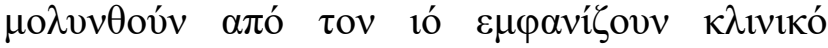

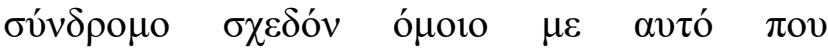

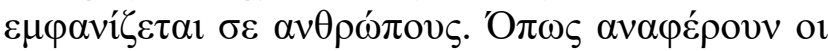

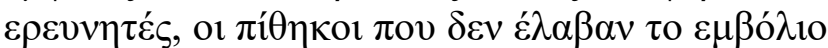

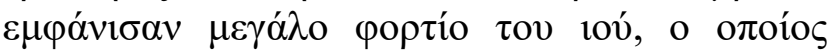

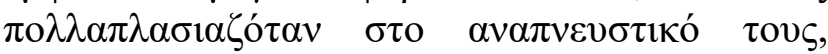

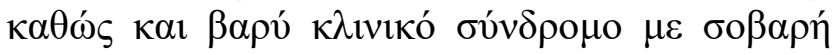

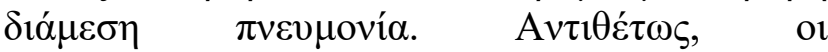

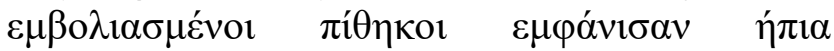

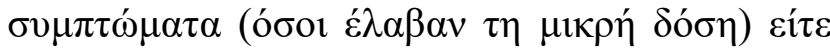

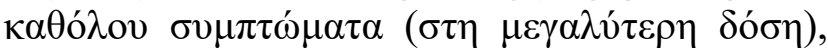

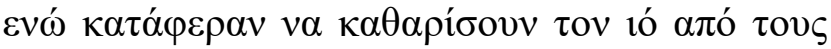

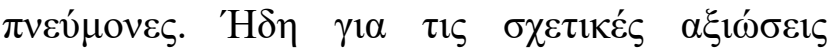

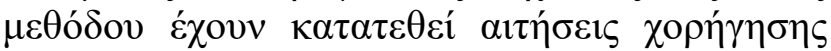

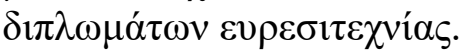

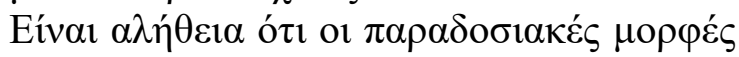

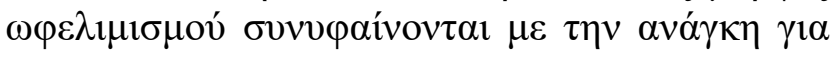

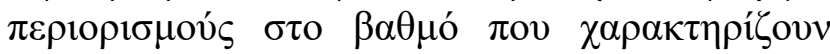

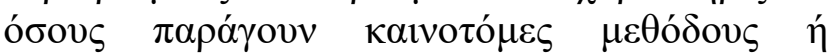

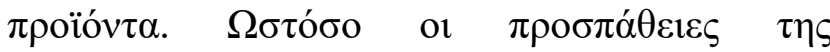

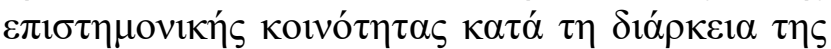

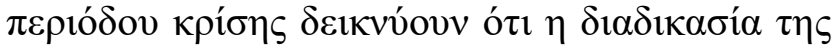

\footnotetext{
${ }^{7} \mathrm{~B} \lambda$. Gao $\mathrm{Q}$ et alia, Development of an inactivated vaccine candidate for SARS-CoV-2, vol. 369, Issue 6499, 2020, 77-81.
} 


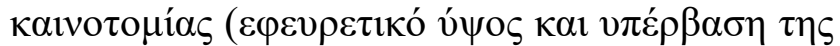

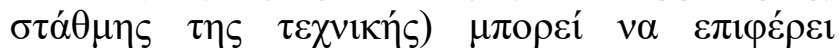

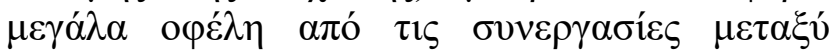

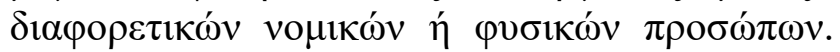

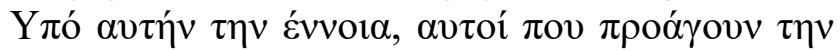

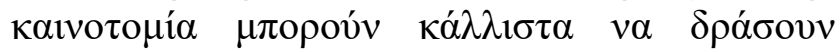

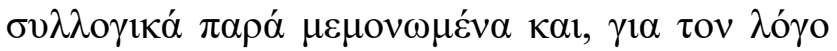

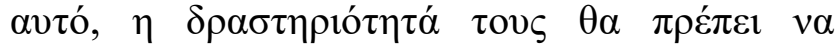

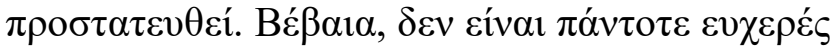

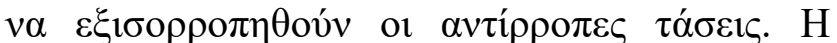

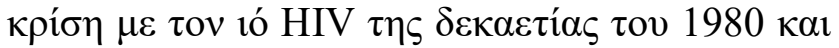

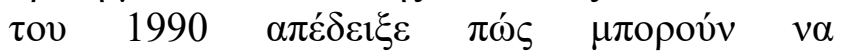

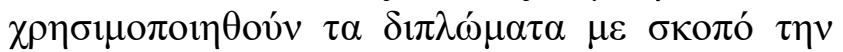

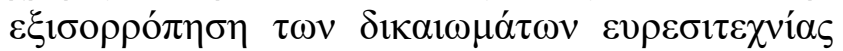

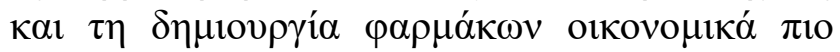

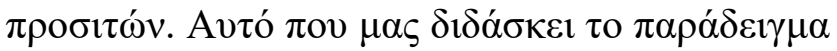

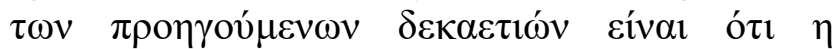

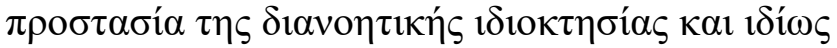

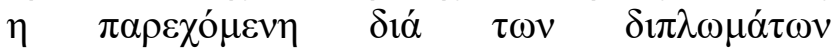

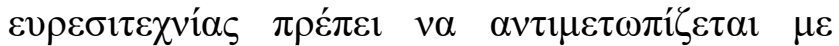

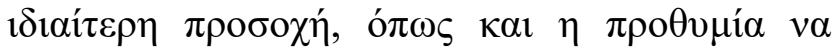

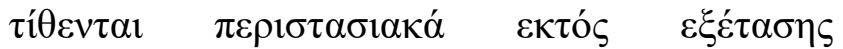

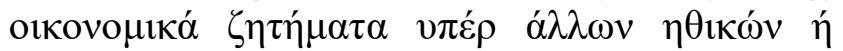

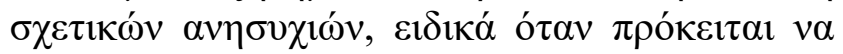

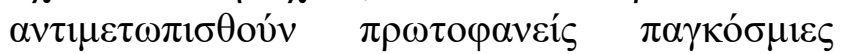

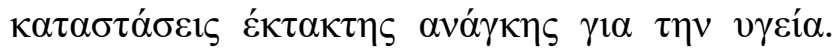

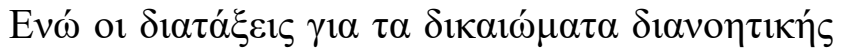

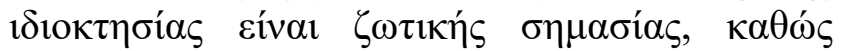

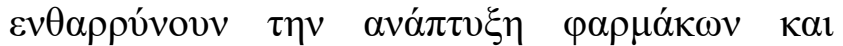

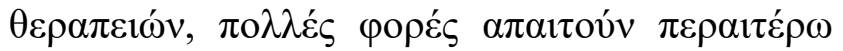
$\pi \rho о \sigma \alpha \rho \mu о \gamma \eta \dot{\eta} \quad \alpha v \alpha \theta \varepsilon \omega ́ \rho \eta \sigma \eta \quad \gamma 1 \alpha \quad v \alpha$ $\varepsilon \xi$

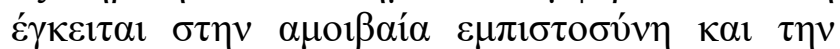
$\pi \alpha \rho \alpha \gamma \omega \gamma \eta \dot{~ \varepsilon 1 \delta i \kappa \eta ́ s ~ v o \mu o \theta \varepsilon \sigma i ́ \alpha \varsigma, ~} \delta \varepsilon \sigma \mu \varepsilon v \tau \imath \kappa \eta ́ s ~ \sigma \varepsilon$

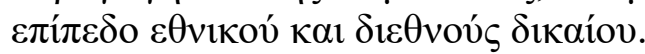

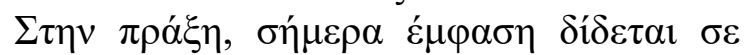

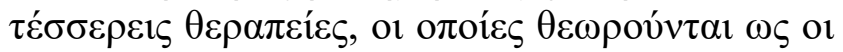

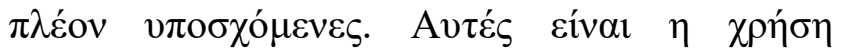

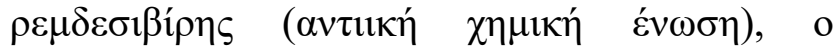

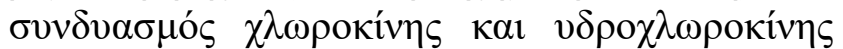

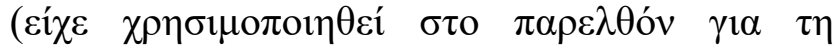

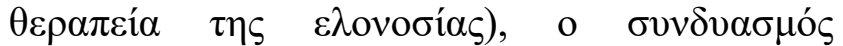

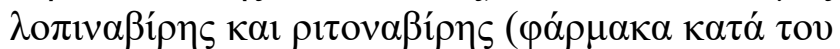

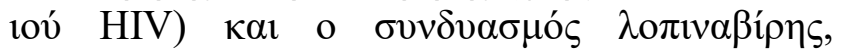



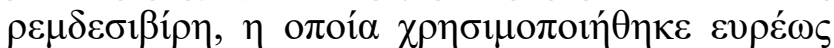

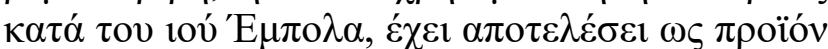

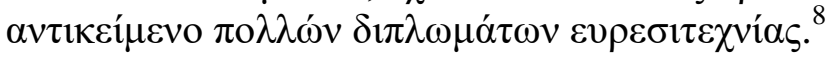

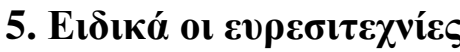

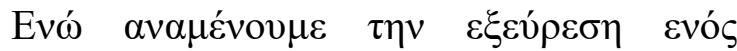

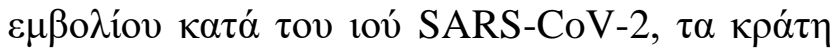

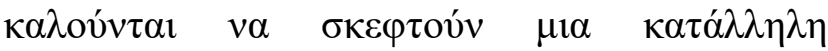

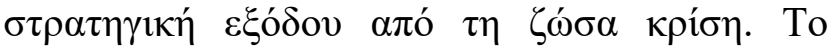

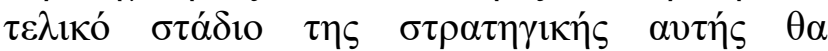

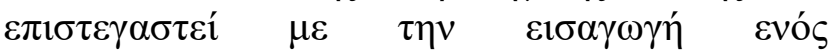

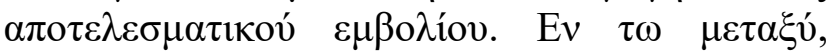

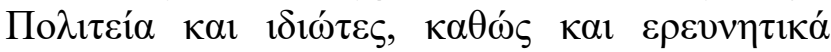

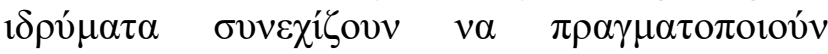
$\mu \varepsilon \gamma \alpha \dot{\lambda} \varepsilon \varsigma \quad \varepsilon \pi \varepsilon v \delta v ́ \sigma \varepsilon 1 \zeta \quad \sigma \tau \eta v \quad \alpha v \alpha \dot{\pi} \tau \tau \xi \eta$

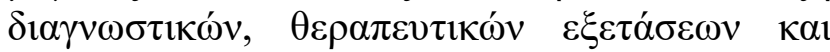

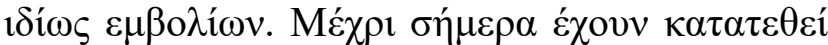

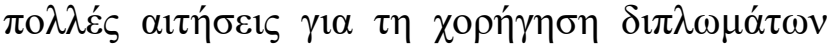

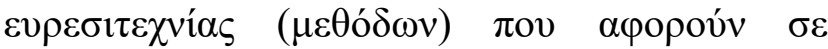

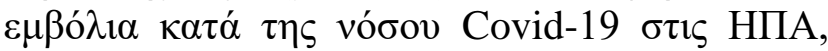

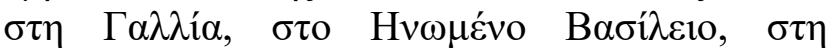

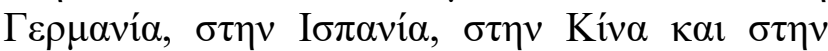

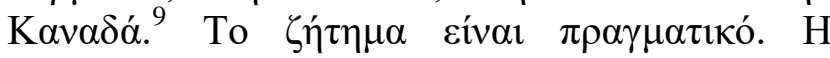

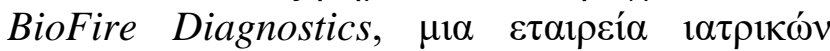

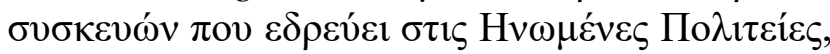

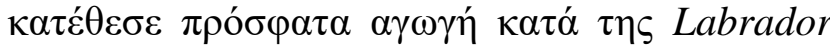
Diagnostics $\quad \gamma 1 \alpha \quad \pi \alpha \rho \alpha \beta i ́ \alpha \sigma \eta \quad \delta \imath \pi \lambda \omega ́ \mu \alpha \tau o \varsigma$

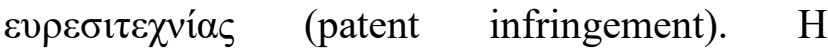

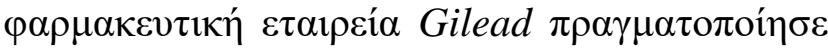

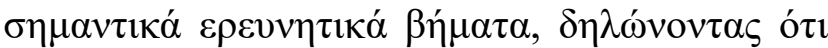

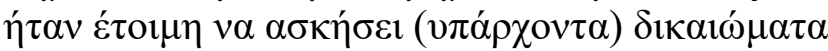

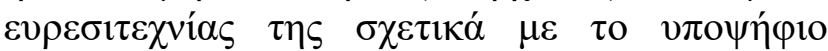

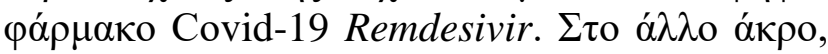

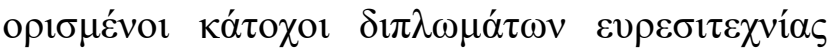

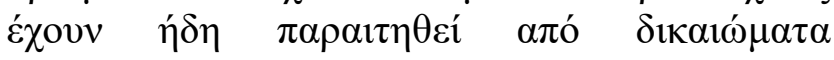

\footnotetext{
${ }^{8} \mathrm{~B} \lambda$. Pilkington S, Remdesivir and the role of patents in tackling Covid-19, $17 \quad$ July 2020. https://news.bloomberglaw.com/pharma-and-lifesciences/insight-remdesivir-and-the-role-of-patents-intackling-covid-19.

${ }^{9} \mathrm{~B} \lambda$.

https://www.timanalytics.eu/TimTechPublic/main.jsp?anal yzer=heatcountry \&dataset $=98764$.
} 


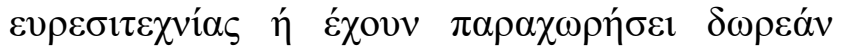

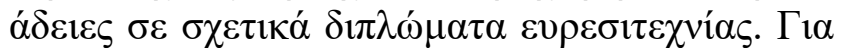

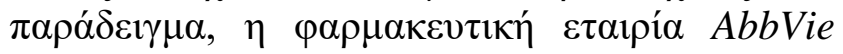
$\pi \alpha \rho \alpha \imath \eta ́ \theta \eta \kappa \varepsilon \quad \alpha \pi o ́ \quad \tau \alpha \quad \delta ı \alpha \iota \omega ́ \mu \alpha \tau \alpha$

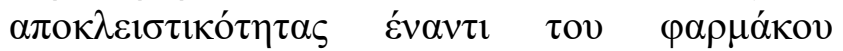
Kaletra $\pi$ ov $\pi \rho 0 \sigma \tau \alpha \tau \varepsilon v ́ \varepsilon \tau \alpha$ al $\alpha \pi$ ó $\delta i ́ \pi \lambda \omega \mu \alpha$

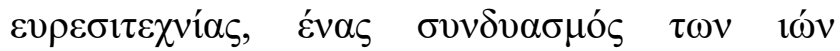

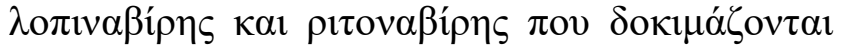

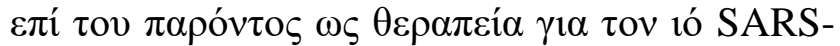
CoV-2.

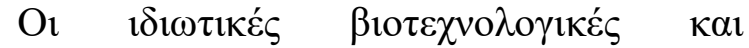

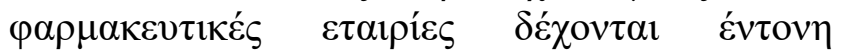

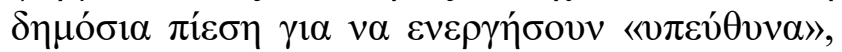

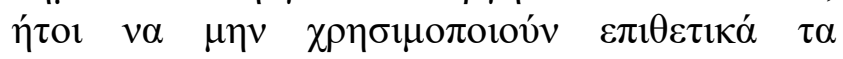

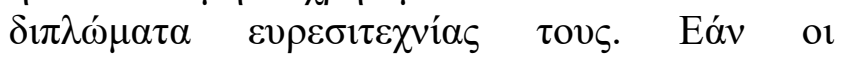

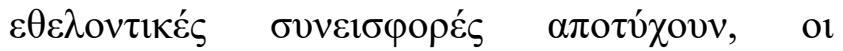

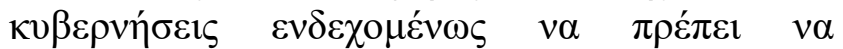

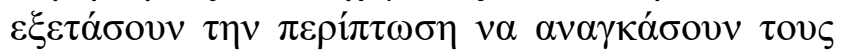

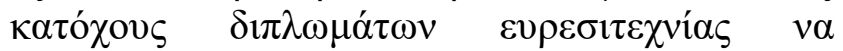

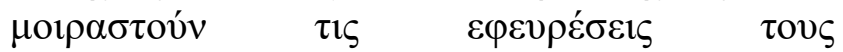

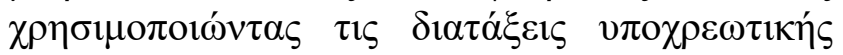

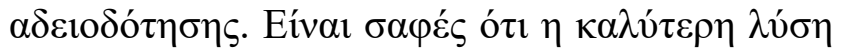

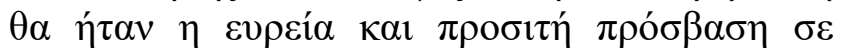

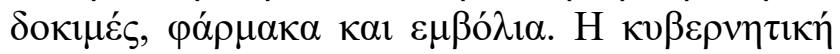

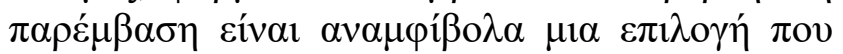

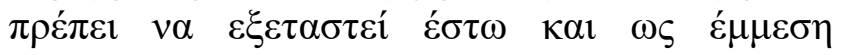

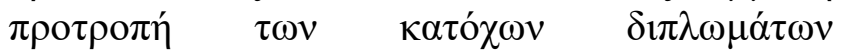

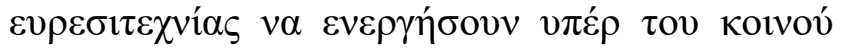

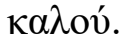

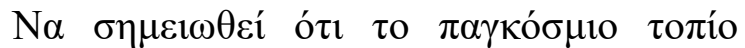

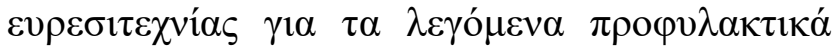

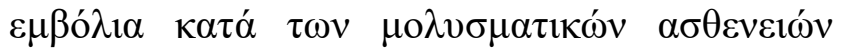

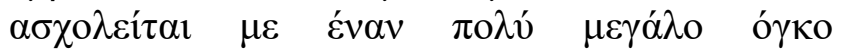

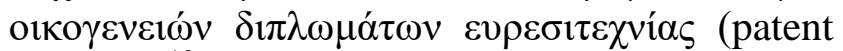

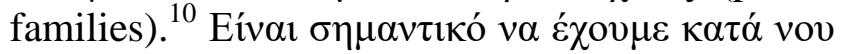

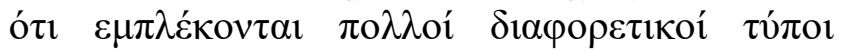

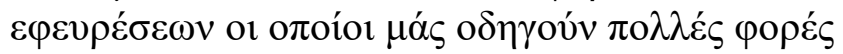

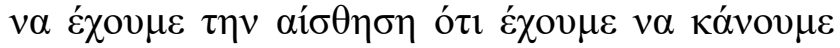

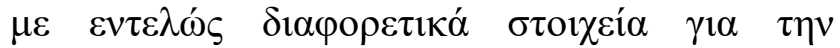

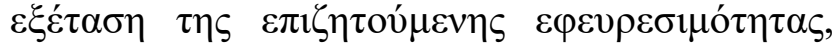

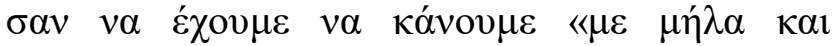

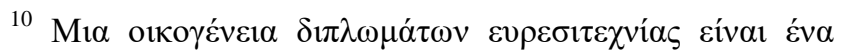

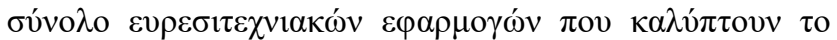
íoı
}

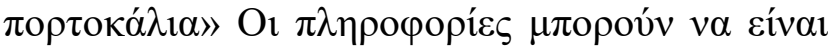

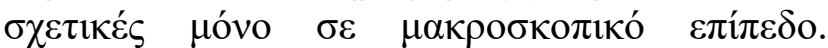

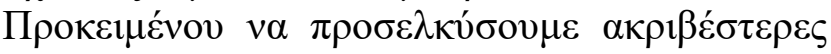

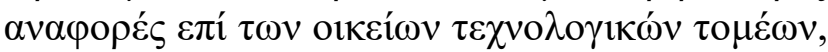
офєі́

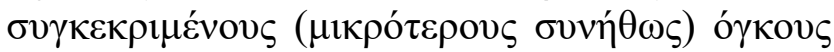

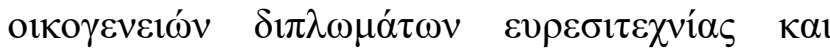

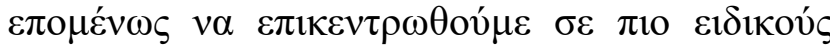

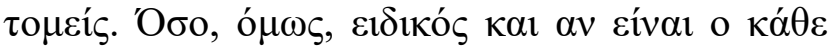

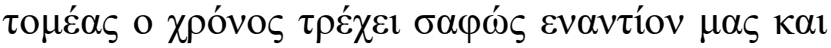

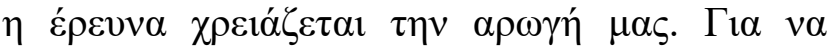

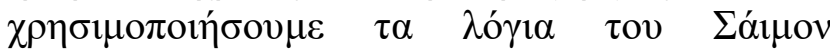

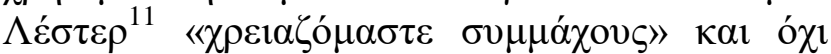

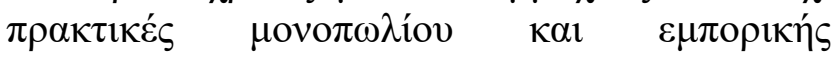

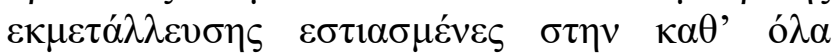

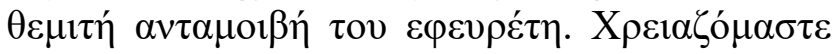

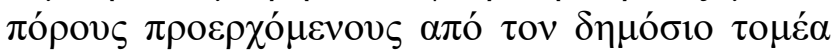

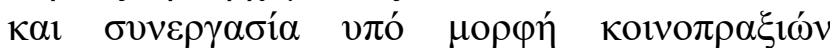
$\varepsilon \kappa \mu \varepsilon \tau \alpha \dot{\lambda} \lambda \lambda \varepsilon v \sigma \eta \varsigma \varepsilon v \rho \varepsilon \sigma \iota \tau \varepsilon \chi v i \omega ́ v$ (patent - pools).

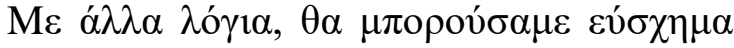

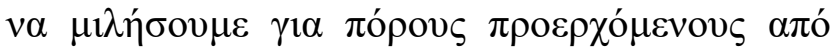

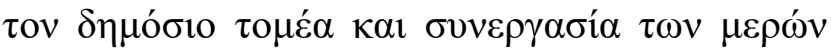

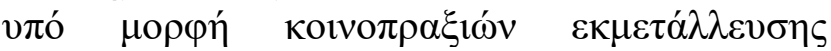

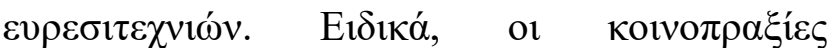

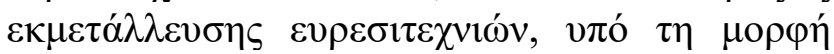

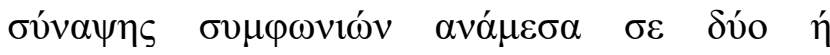
$\pi \varepsilon p 1 \sigma \sigma o ́ \tau \varepsilon \rho o v \varsigma \quad \kappa \alpha \tau o ́ \chi 0 v \varsigma \quad \delta i \pi \lambda \omega ́ \mu \alpha \tau o \varsigma$

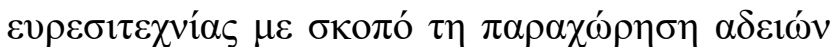

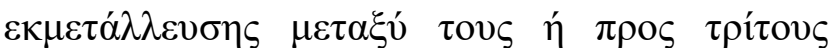

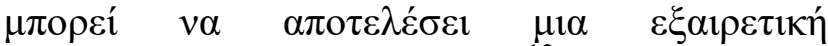

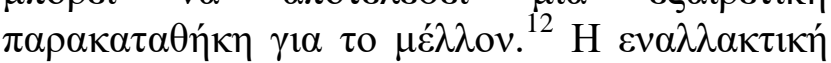

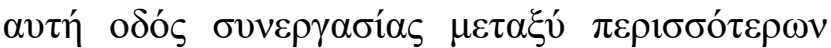

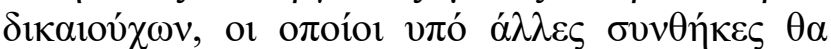

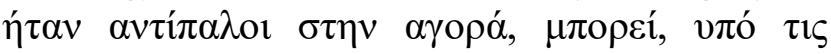

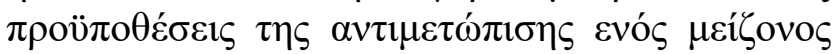

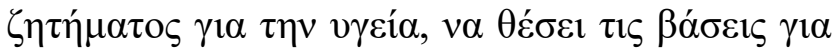

\footnotetext{
${ }^{11} \mathrm{~B} \lambda$. Lester S, We Need a Coronavirus Vaccine - Patents Might Slow the Process, 2020. https://www.cato.org/publications/commentary/we-needcoronavirus-vaccine-patents-might-slow-process.

12 Hovenkamp E, Hovenkamp HJ, Patent Pools and Related Technology Sharing, $\sigma \varepsilon$ Blair RD, Sokol DD (eds.) Cambridge Handbook of Antitrust, Intellectual Property and High Tech, Cambridge University Press, 2017, 358-376.
} 


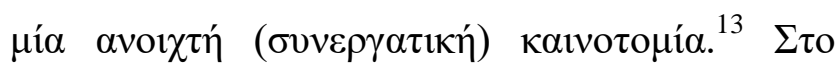

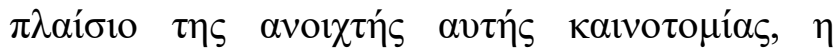

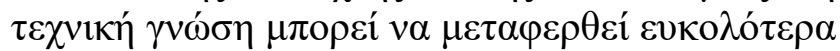

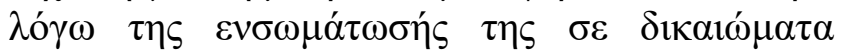

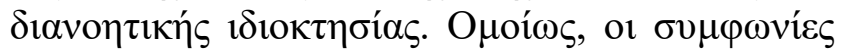

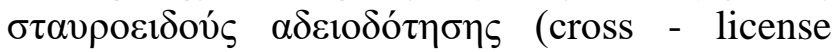

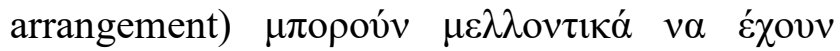

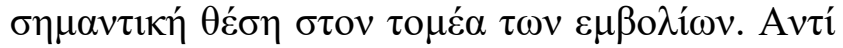

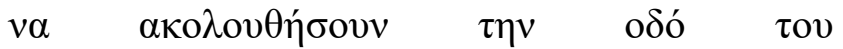

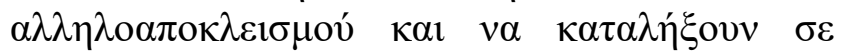

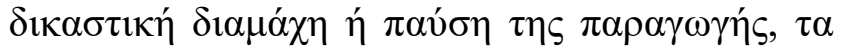

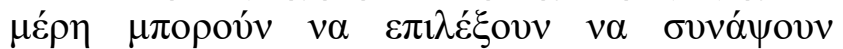

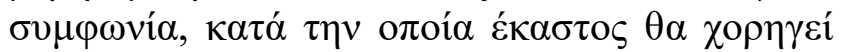

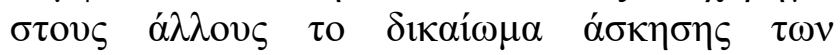

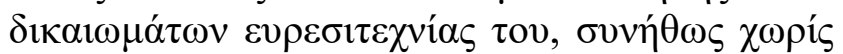

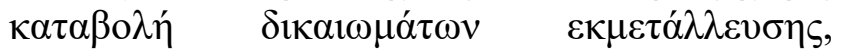

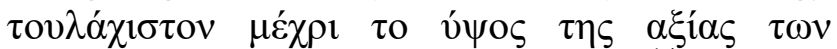

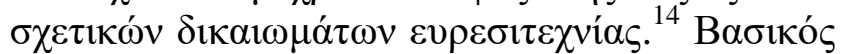

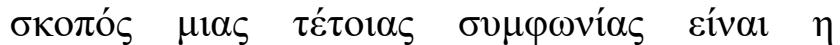

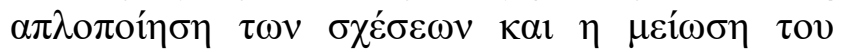

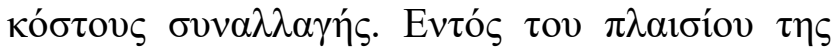

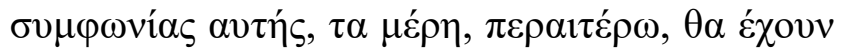

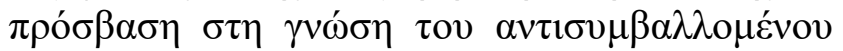

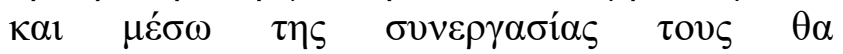

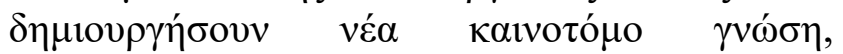

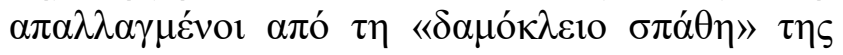

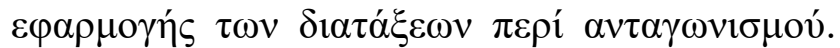

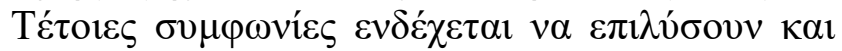

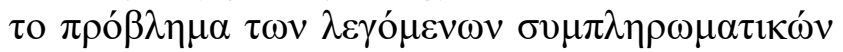

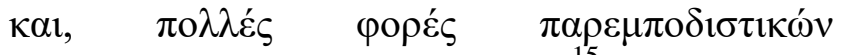
$\varepsilon v \rho \varepsilon \sigma i \varepsilon \chi \chi \vee \omega ́ v$ (blocking patents). ${ }^{15}$

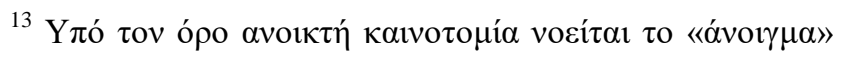

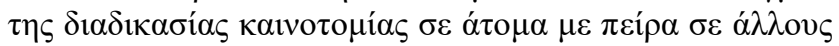

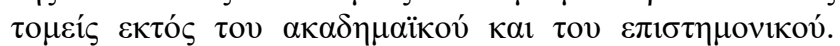

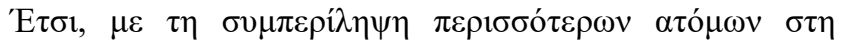

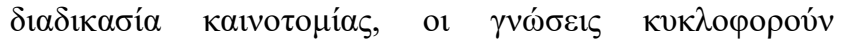

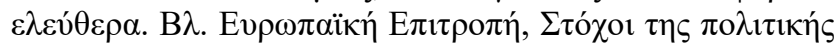

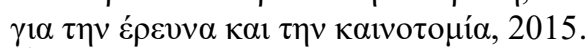

14 Armillotta M, Technology Pooling Licensing Agreements: Promoting Patent Access Through Collaborative IP Mechanisms, Munich: Nomos Verlagsgesellschaft mbH, 2010, 30.

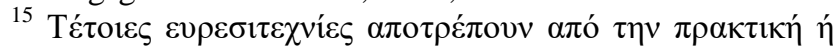

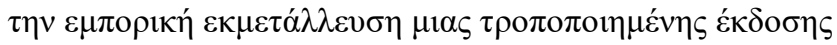

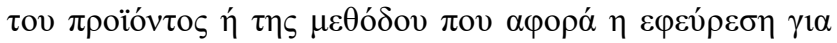

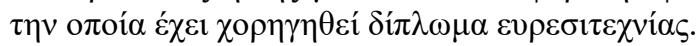

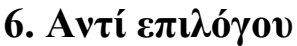

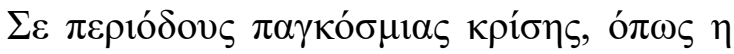

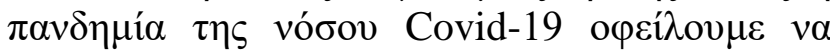

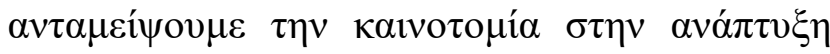

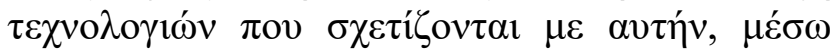

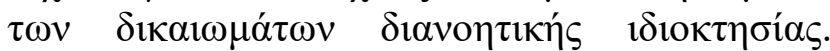

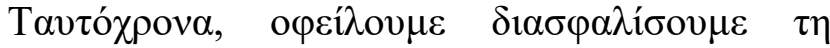

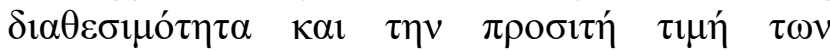

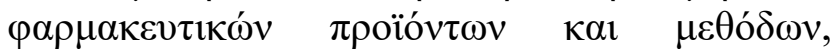

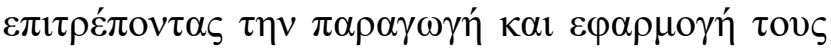

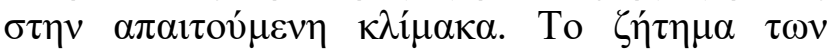

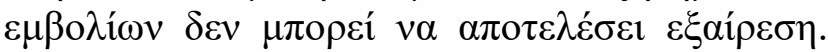

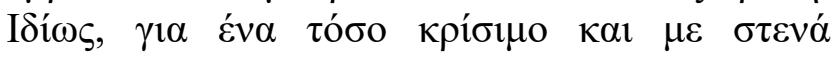

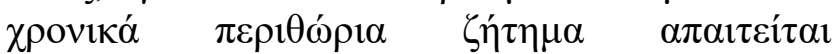

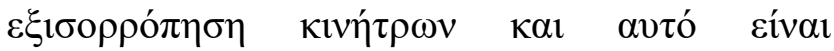

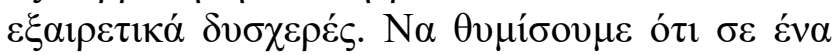

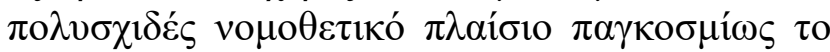

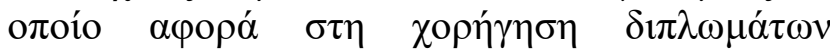

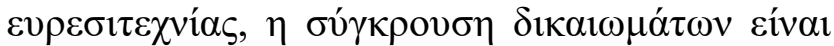

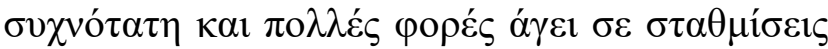

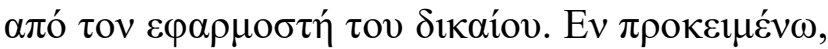

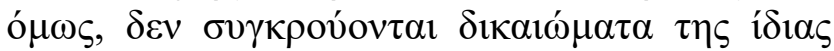

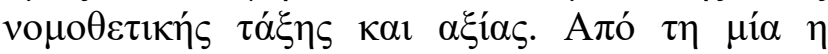

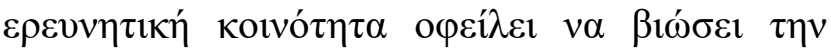

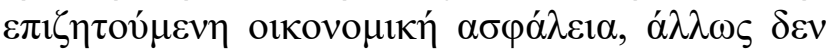

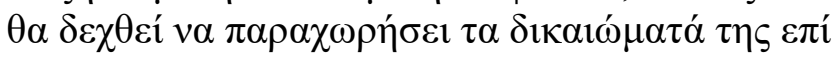

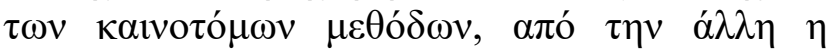

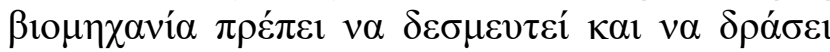

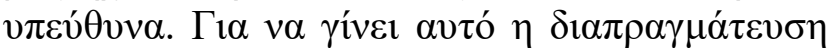

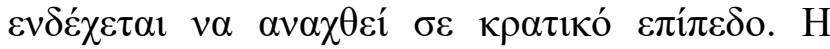

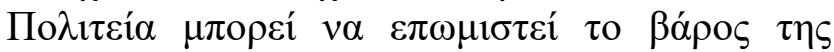

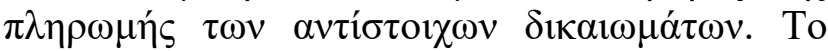

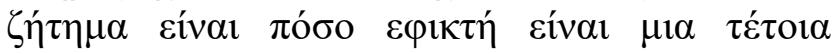

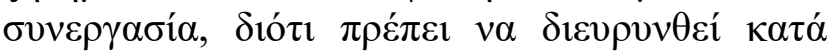

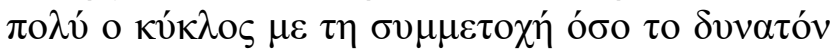

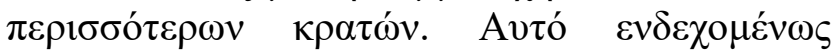

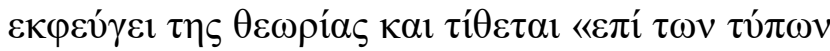

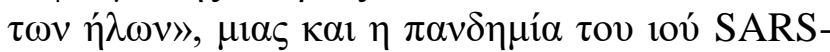

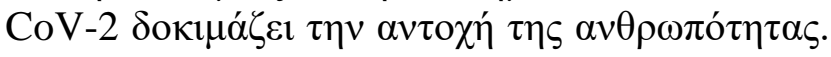

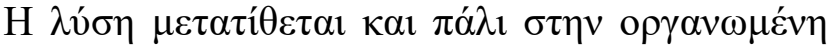

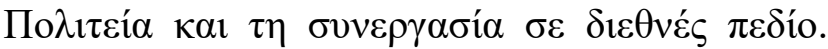

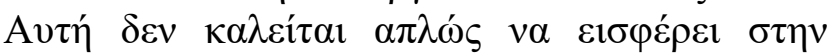

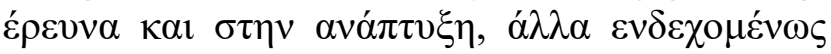

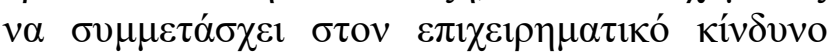

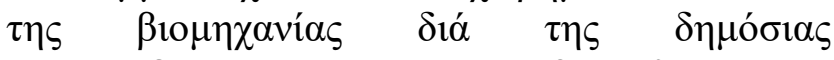

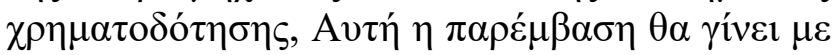

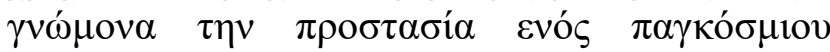

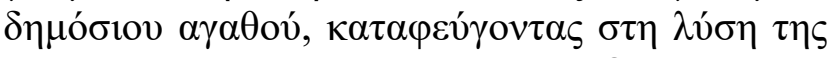

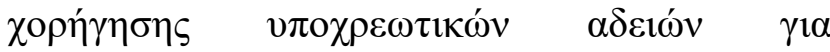




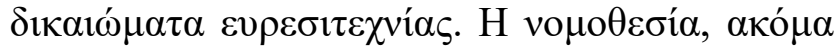

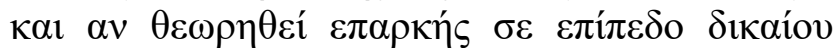

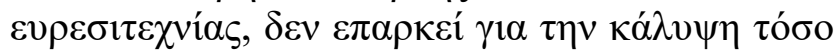

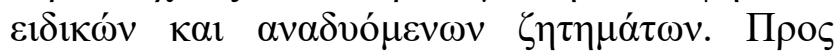

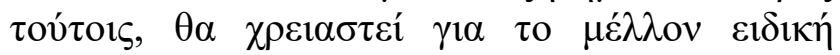

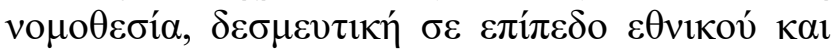

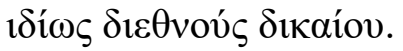

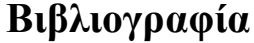

Abbott F, The TRIPs Agreement Article 73 Security Exceptions and the COVID-19 Pandemic, Research Paper 116, South Centre, 2020, 7.

Armillotta M, Technology Pooling Licensing Agreements: Promoting Patent Access Through Collaborative IP Mechanisms, Munich: Nomos Verlagsgesellschaft mbH, 2010, 30.

Bofinger P, Dullien S, Felbermayr G, Fuest C, Hüther M, Südekum J, Weder di Mauro B, Economic implications of the COVID-19 crisis for Germany and economic policy measures, $\sigma \varepsilon$ Baldwin R, Weder di Mauro B (eds), Mitigating the COVID economic crisis: Act fast and do whatever it takes, a VoxEU.org eBook, CEPR Press, 2020.

Branswell $\mathrm{H}$, As coronavirus pandemic worsens, health officials fear nationalization of drugs and supplies, Stat Health, 15 March 2020.
Foray D, de Rassenfosse G et alia, COVID-19: Insights from Innovation Economists, 2020. https://papers.ssrn.com/sol3/papers.cfm?abstract _id=3575824.

Gao Q et alia, Development of an inactivated vaccine candidate for SARS-CoV-2, vol. 369, Issue 6499, 2020, 77-81.

González OB, Thinking slow about IP in times of Pandemic, IIC Int Rev Ind Prop Copyr Law, 25 May 2020, 1-4.

Hovenkamp E, Hovenkamp HJ, Patent Pools and Related Technology Sharing, $\sigma \varepsilon$ Blair RD, Sokol DD (eds.) Cambridge Handbook of Antitrust, Intellectual Property and High Tech, Cambridge University Press, 2017, 358-376.

Lester S, We Need a Coronavirus Vaccine Patents Might Slow the Process, 2020. https://www.cato.org/publications/commentary/ we-need-coronavirus-vaccine-patents-mightslow-process.

Srinivas KR, Intellectual Property Rights and Innovation in the Times of Corona Epidemic, RIS Policy Brief, no. 89, 2 April 2020. 